\title{
Modified Tapered Slot-line Antennas for Special Applications
}

\author{
Wen-Xun Zhang \\ State Key Lab. of Millimeter Waves, Southeast University, China \\ Correspondence: Wen-Xun Zhang, wxzhang@ieee.org \\ Manuscript communication: received 16 September 2012, accepted 5 January 2013
}

\begin{abstract}
Tapered slot-line antenna (TSA) is a well known printed end-fire antenna with traveling-wave feature. In order to further improve its performances and extend its functions, several techniques with patents were developed in SEU, and summarized in this article. Here include: grating-loaded TSA for gain enhancement, CPW back-fed TSA for broadening impedance bandwidth, asymmetric TSA structure for beam shaping and optimization, coupled TSA with hybrid network for monopulse beam forming. Both conceptual illumination and simulated/measured results are described.
\end{abstract}

Keywords- Tapered slot-line antenna, gain enhancement, metal-grating loading, bandwidth broadening, UWB feeding, beam shaping, dual-band antenna, monopulse-beam duplexer

This work was partly published in several different media individually and summarized in presentation to the 2012 International Conference on Advanced Technologies for Communications (ATC), Hanoi, Vietnam.

\section{INTRODUCTION}

Recently, miscellaneous printed antennas have been widely applied in various $\mathrm{RF} /$ microwave equipments or systems, from wireless communication to phasedarray radar, from satellite telemetry to radio-frequency identification, from electronic counter-measures to embedded bio-electronic chip, etc. This enormous family can be classified into three branches according to their different radiation mechanisms: (1) resonant antennas as dipole, loop, slot, patch, etc. (2) traveling-wave antennas as tapered slot-line, meander-line, grating, etc. (3) optical antennas as reflect-array, transmit-array, compound air-fed array, etc. This paper is focusing to some new development of the tapered slot-line antenna (TSA).

The original structures of TSA were proposed by P.J. Gibson [1] and S.N. Prasad et al. [2] to the same forum of the 9th European Microwave Conference by using individual terminology and configuration as shown in Figure 1. Here both Vivaldi antenna and Linear-TSA are simply called as TSA.

In general, the slot-line is terminated by a backward short-circuit stub, and fed by a crossed microstrip-line which printed on other side of dielectric substrate with an open-circuit stub; as shown in Figure 2, those resonated stubs always restrict the impedance bandwidth of antenna. Hence, the feeding scheme will be a key technique of bandwidth enhancement.

These traditional TSAs possess common features as: traveling-wave radiation with multi-octave bandwidth; end-fire pattern with moderate directive gain and obvious side- lobes; printed structure with small cross-section; and also integrating compatibility with devices or circuits. However, their performances are

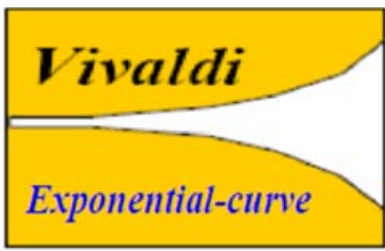

(a) Vivaldi Antenna

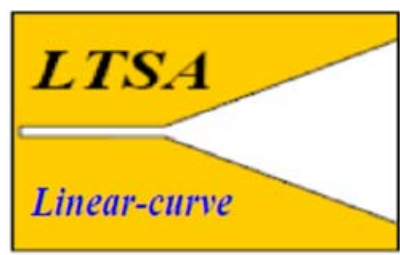

(b) Linear TSA
Figure 1. Original structures of tapered slot-line antenna.

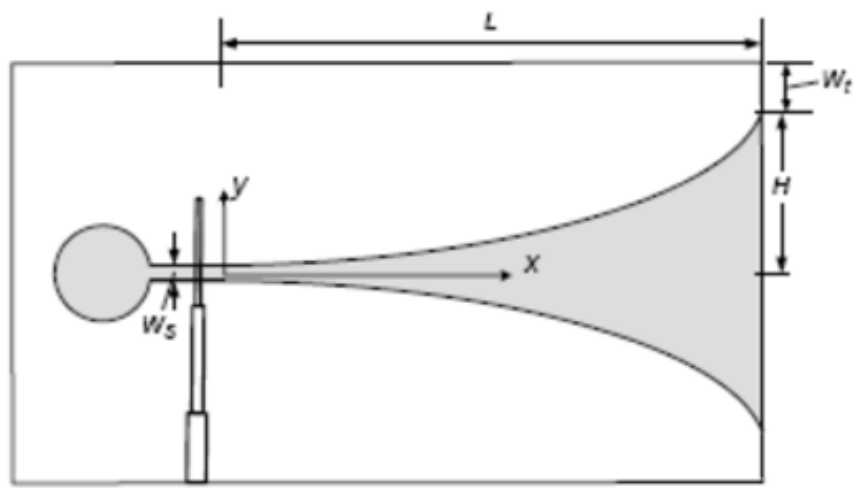

Figure 2. Traditional feeding structure with microstrip-line and slotline stubs.

waiting to be improved for gain enhancement, side-lobe suppression, beam-width equality in E- \& H- planes, and broadening impedance bandwidth. In addition, the possibility for extending the functions of TSA is expected.

In principle, some possible confused concepts on TSA should be clearly discriminated as follows:

1) TSA is neither a 'tapered slot antenna' chiseled on a conductive screen nor a 'tapered slot-line' as a pure transmission line; but the transmission and 
radiation of TSA simultaneously betide on a slotline with tapered slot-width.

2) TSA is different from a 'traveling-wave wire antenna' whose radiation is contributed by the currents along a pair of wires only; but all the currents distributed on a pair of metallic plates as shown in Figure 3 contribute to the radiation of TSA.

3) TSA possesses a longitudinal radiated aperture; it could not be named as a 'planar horn antenna' whose transversal aperture results in broadside radiation.

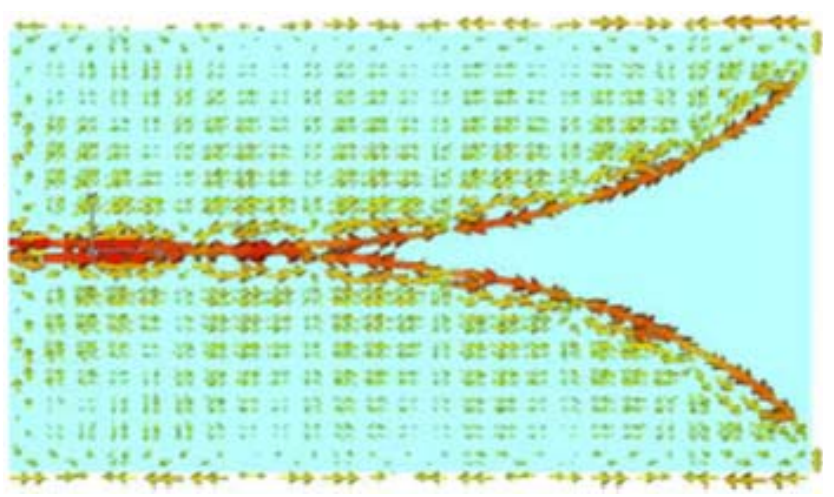

Figure 3. Currents distribution on whole plate of TSA.

\section{Improvement of Traditional TSA}

For comparing the main performances between Vivaldi antenna and Linear-TSA (LTSA) based on the same sizes $84 \times 54 \mathrm{~mm}$ of substrate and traditional feeding, their typical frequency response curves of both $\left|S_{11}\right|$ and gain are simulated (excluding feed structure) as shown in Figure 4. Where (a) (b) show that Vivaldi antenna has slightly wider bandwidth from $f_{\min }=12.15$ $\mathrm{GHz}$ than LTSA from $f_{\min }=12.80 \mathrm{GHz}$ for $\left|S_{11}\right| \leq$ $-10 \mathrm{~dB}$; but (c) shows that gain rise vs. frequency started from $13.5 \mathrm{dBi}$ (at $12.2 \mathrm{GHz}$ ) up to $15.9 \mathrm{dBi}$ (at $18 \mathrm{GHz}$ ) for LTSA, it is faster than from $13.5 \mathrm{dBi}$ to $14.1 \mathrm{dBi}$ for Vivaldi antenna; also the cross-polar-level of Vivaldi antenna $(X-P \leq-33 \mathrm{~dB})$ is slightly lower than that of LTSA $(X-P \leq-31 \mathrm{~dB})$. In above TSA samples, to perform higher gain must increase electric length $(L / \lambda)$ of antenna, so it usually suits in higher SHF band rather than lower SHF even UHF/ VHF bands.

\subsection{Gain Enhancement}

In order to enhance the gain for relatively shorter TSA, a scheme by loading metal-strip grating as additional director imbedded into the slot of LTSA (Figure 5) [3] is developed. Where the traditional LTSA of sizes $84 \times 54 \mathrm{~mm}$ without directive grating provides about $8 \mathrm{dBi}$ peak gain with stable frequency response and about $\sim 25 \%$ practical bandwidth for joint gainimpedance as $\left|S_{11}\right| \leq-12.5 \mathrm{~dB}$.

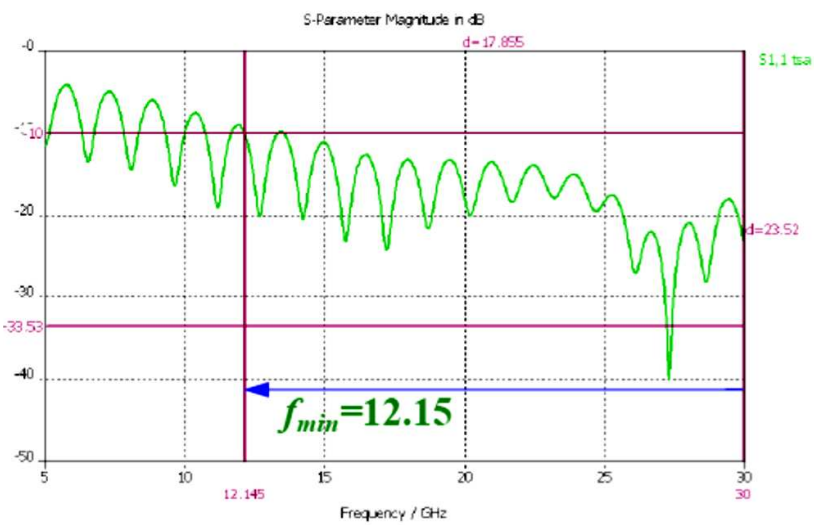

(a) Curve of $\left|S_{11}\right|$ for Vivaldi antenna

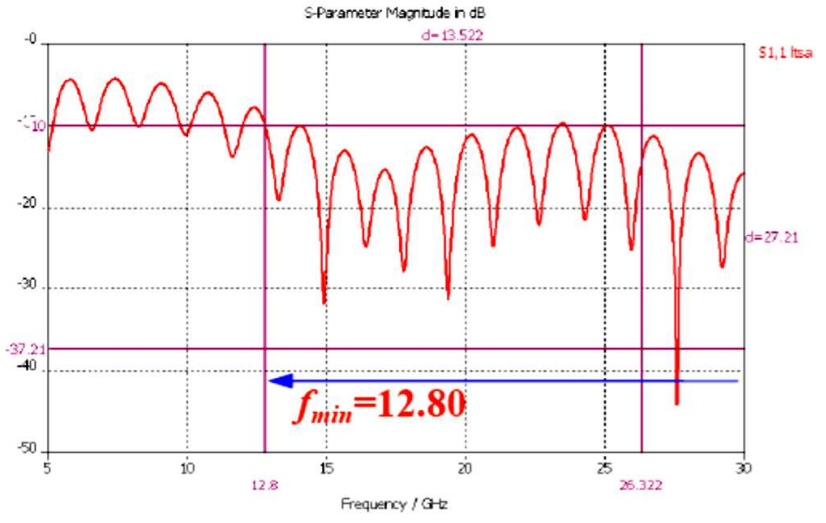

(b) Curve of $\left|S_{11}\right|$ for LTSA

Eroustond gan 3 d

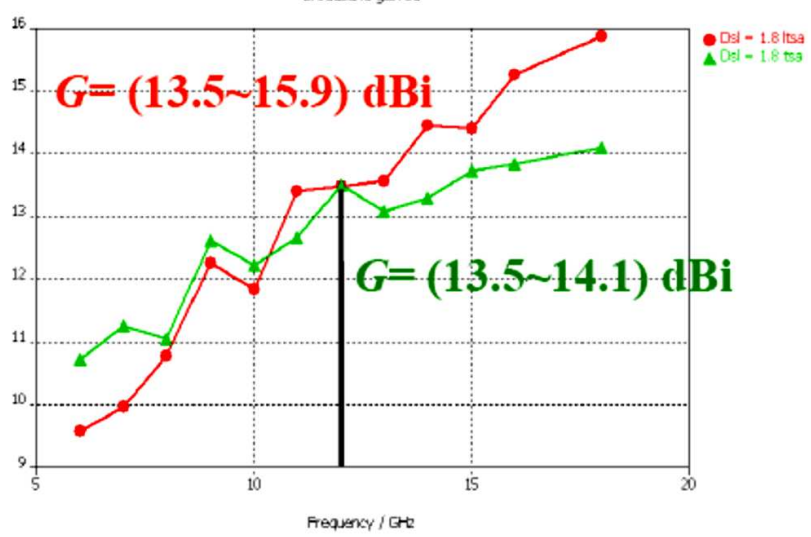

(c) Curves of gain for Vivaldi antenna \& LTSA

Figure 4. Comparison of frequency responses between Vivaldi antenna \& LTSA.

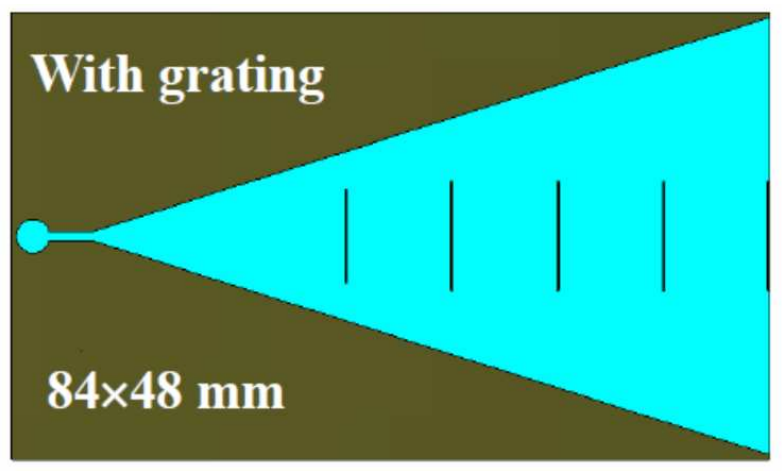

Figure 5. LTSA loaded by metal-strip grating. 


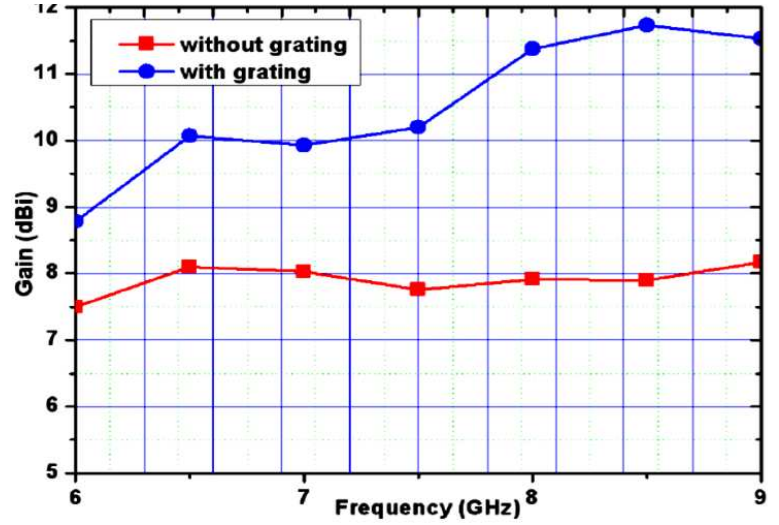

(a) Curves of gain

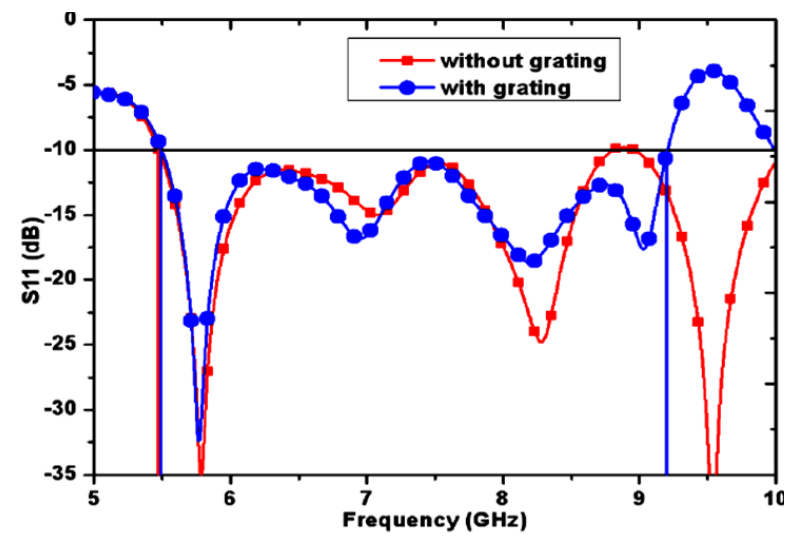

(b) Curves of $\left|S_{11}\right|$

Figure 6. Comparison of frequency responses without \& with grating.

Usually, the cost to enhance the peak gain must be obviously extending electrically length and width of the antenna. Whereas the LTSA with the same sizes and loaded by directive grating improves the performances as: $11.7 \mathrm{dBi}$ peak gain with $3.7 \mathrm{~dB}$ enhancement at 8.5 $\mathrm{GHz}$; thus $42 \%(6 \sim 9.2 \mathrm{GHz})$ practical bandwidth for both $V S W R \leq 2: 1$ (for $f_{\max }$ ) and $G_{\text {drop }} \leq 3 \mathrm{~dB}$ (for $\left.f_{\min }\right)$ as shown in Figure 6.

\subsection{Side-lobe Suppression}

In order to suppress the side/back-lobes produced mainly by edge currents, to etch slots acting as chokes on the side-/back-edges (Figure 7) is effective, while $S L L$ can be reduced about $2 \sim 4 \mathrm{~dB}$.

In addition, a metal back-plate with appropriate sizes perpendicularly assemble to the TSA sheet can also effectively suppress the back-lobe-level.

\section{TSA with UWB FeEding SRUcture}

Though the traveling-wave mechanism provides wideband properties for both radiation pattern and impedance matching, and the frequency coverage of TSA with enough electrical-length can approach to $6: 1$ [4]; however, the microstrip-line stub (open-circuit) and the slot-line stub (short-circuit) in feeding structure always result in bandwidth limitation of entire TSA. In order to adequately dredge up the ultra-wide-band

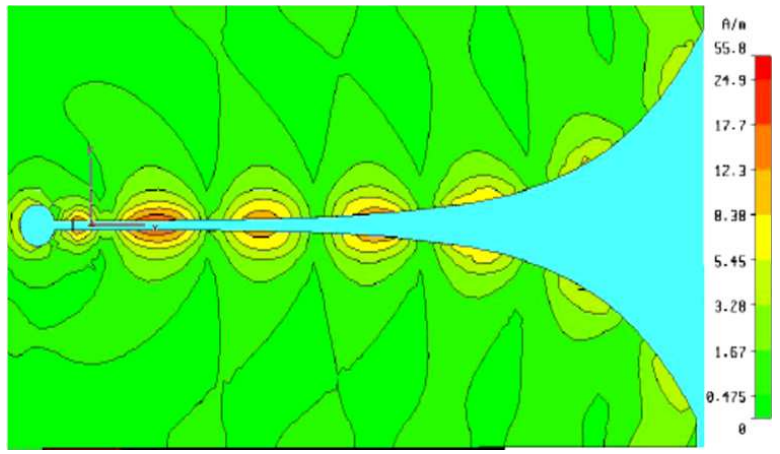

(a) Without side/back slots

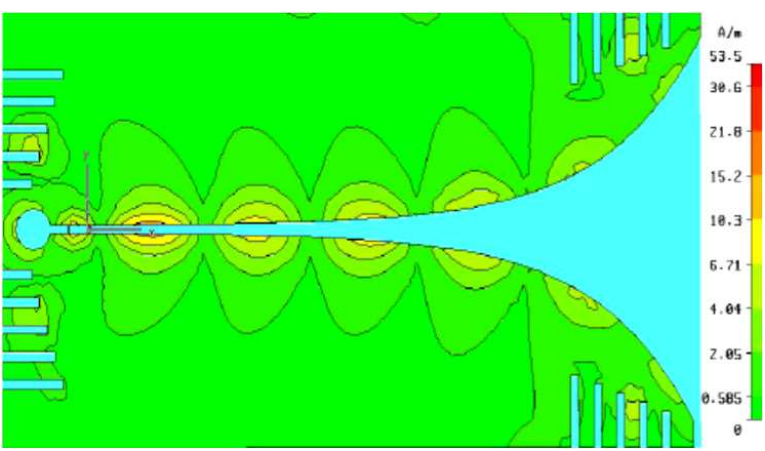

(b) With side/back slots

Figure 7. Current distribution on TSA.

(UWB) feature of TSA, an improved design was developed [5] by employing bilateral TSA (B-TSA) and a coated dielectric back-plate cut with a П-type slot as shown in Figure 8. Where a pair of upper-half B-TSA connect to the outside part of $\Pi$-type slot as a ground plane; another pair of lower-half B-TSA connect to the inside part of $\Pi$-type slot as a central strip. Then the combination of these ground plane and central strip on the back-plate just form an unbalanced coplanar waveguide (CPW), which directly feeds to the balanced B-TSA without frequency sensitivity.

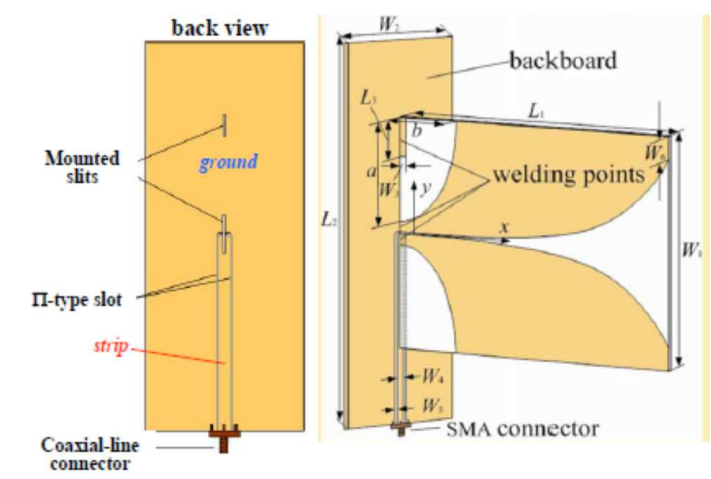

Figure 8. Structural sketch of UWB CPW-fed bilateral TSA.

A prototype with sizes of $93 \times 96 \mathrm{~mm}$ for B-TSA and $40 \times 100 \mathrm{~mm}$ for back-plate was fabricated, then its simulated results verified by measured data provide both $V S W R \leq 2: 1$ and $G \geq 6 \mathrm{dBi}$ on practical bandwidth of $4 \sim 16 \mathrm{GHz}$ (i.e. $4: 1$ frequency coverage) as shown in Figure 9. 


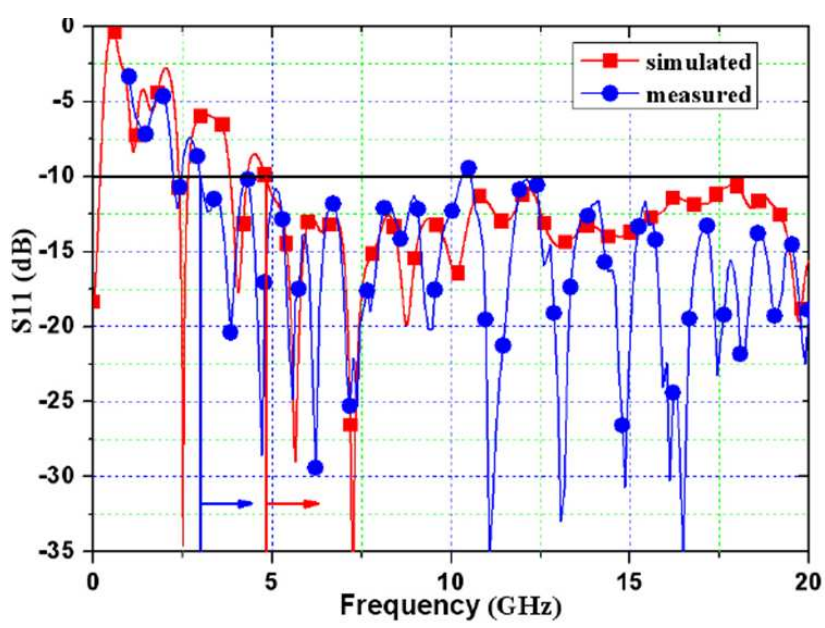

(a) Curves of $\left|S_{11}\right|$

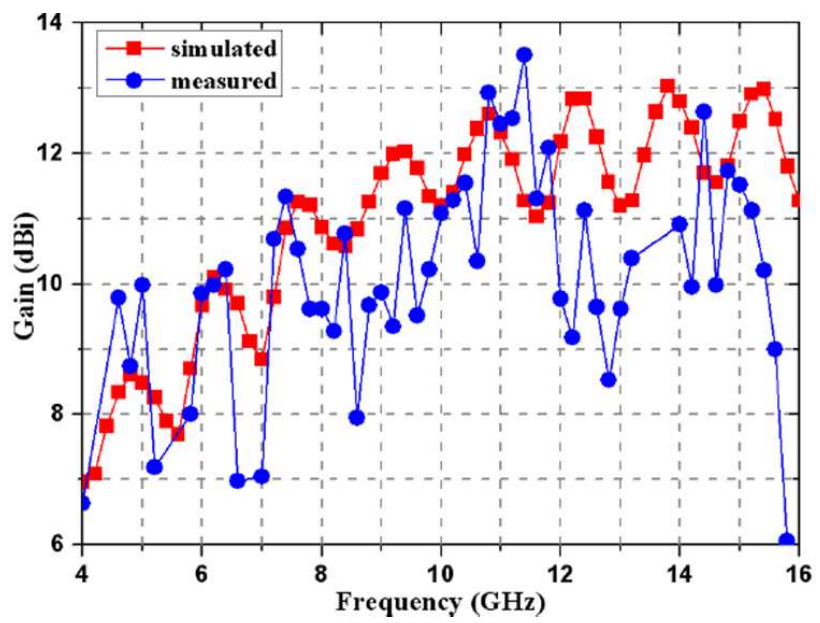

(b) Curves of gain

Figure 9. Frequency responses of UWB-fed bilateral TSA

\section{BeAm-Shaped TSA Used in Base-Station}

Normally, a TSA of symmetric structure with respect to its axis radiates symmetric beam; accordingly, an asymmetric TSA (A-TSA) will radiate asymmetric beam too. The latter may be utilized for a base-station of mobile communication service, to achieve a vertical pattern with uniform coverage without null in downward radiation and appropriately reduced upward radiation. While the parameter of directive gain becomes senseless, but a new parameter called as coverage efficiency $\eta_{c}$ may be adopted as:

$$
\eta_{c}=\frac{P_{r[\text { downward }]}}{P_{r[\text { total }]}} \times 100 \%
$$

where $P_{r}$ is radiation power. Obviously, $\eta_{c}$ must be $\geq$ $50 \%$.

Firstly, a scaled prototype with $70 \times 46 \mathrm{~mm}$ of A-TSA and $30 \times 52 \mathrm{~mm}$ of back-plate was designed and fabricated [6] as shown in Figure 10. It performs: frequency coverage $3: 1(5.56 \sim 16.8 \mathrm{GHz})$ for $V S W R \leq 2: 1$; bandwidth $12 \%(9.5 \sim 10.7 \mathrm{GHz})$ for $G \geq 11.2 \mathrm{dBi}$ with $4^{\circ}$ tilt-down beam; $\eta_{c} \geq 60 \%$; and HPBW (half-powerbeam-width) $\approx 65 \%$ in horizontal plane. It satisfies most specification of base-station antenna, except the

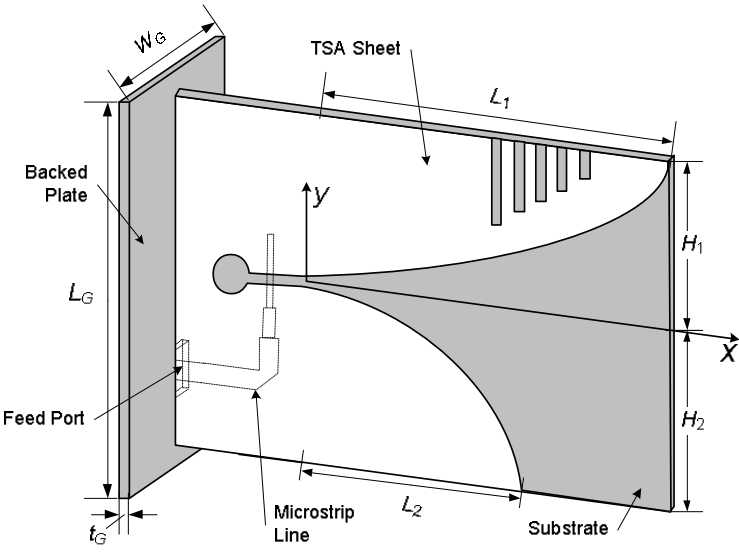

Figure 10. Asymmetric TSA for base-station .

gain is not high enough due to the uniform coverage service. The main advantages of this A-TSA is only one element with single feed point; avoids complicated feed network with many power dividers, transmission bends and connectors, which always result in power loss, bandwidth restriction and potential inter-modulation among signal channels. Figure 11 shows its typical patterns in both vertical and horizontal planes.

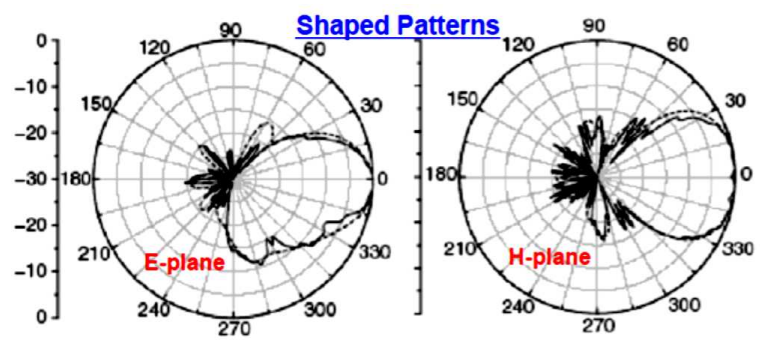

Figure 11. Typical patterns of asymmetric TSA.

Secondary, a further prototype for dual-band WIMAX application with $210 \times 120 \mathrm{~mm}$ of A-TSA and $40 \times 120 \mathrm{~mm}$ of double-layer back-plates was designed and fabricated [7] as shown in Figure 12.

In which, a set of tilted metal-strips grating is employed for coordinating the impedance matching and coverage efficiency. For the former, the bandwidth for $V S W R \leq 2: 1$ is quite wide as $(2.0 \sim 6.0 \mathrm{GHz})$ in measurement; but for $V S W R \leq 1.5: 1$ in simulation covers two separated WIMAX bands as lower $(3.3 \sim$ 3.8) $\mathrm{GHz}$ and higher $(5.1 \sim 5.8) \mathrm{GHz}$ bands. For the latter, the coverage efficiency $\eta_{c} \geq 69.5 \% \& 73.2 \%$, and $G=(9.2 \sim 9,7) \mathrm{dBi} \&(8.5 \sim 10.5) \mathrm{dBi}$, respectively, for lower \& higher bands. Besides, the beam have $(8 \sim 12)^{\circ}$ tilt-down in vertical plane.

In addition, the slots etched along the upper sideedge suppress the upward radiation; and the doublelayer back-plates forms a choke at the side of backplates for suppressing the back-lobe level under $-20 \mathrm{~dB}$. 


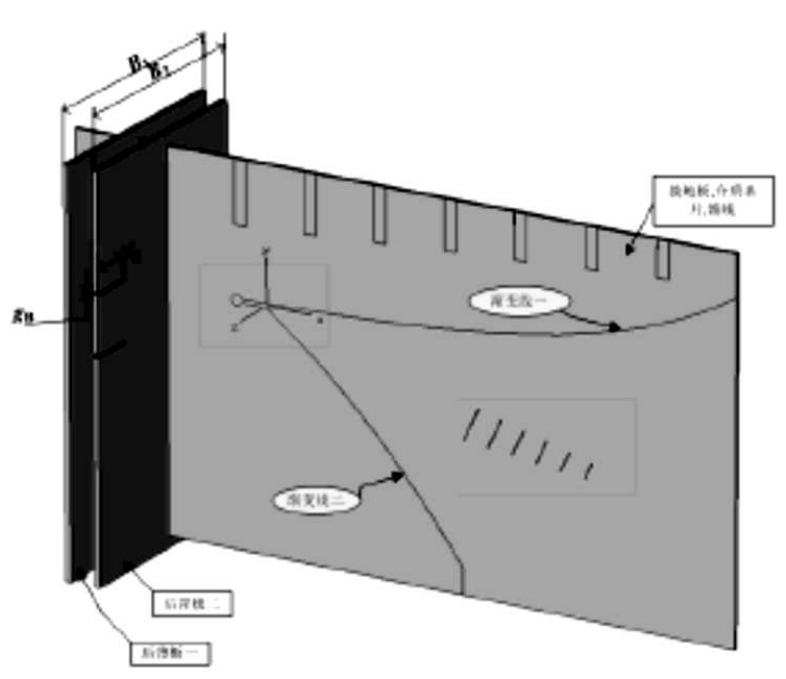

(a) Sketch

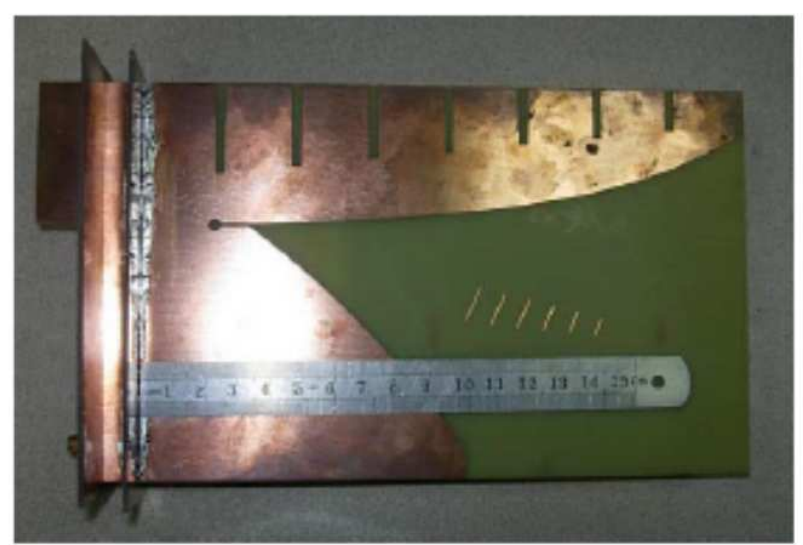

(b) Photo

Figure 12. Dual-band Asymmetric TSA for WIMAX.

\section{Beam-Shaped TSA As A Feed of Air-Fed ARRAY}

By means of wideband property, the TSA had been popularly applied alone as a reference in antenna test, or a receptor in electronic counter-measuring; or the element of phased-array. However, it can also be used as a feed of air-fed array antenna, while a saddle-shaped beam is required for achieving uniform amplitude distribution on aperture within the illuminated angle of the feed, and sharply slope-down outside the angle.

A radiation pattern with symmetric saddle-shaped beam possesses a pair of peaks out-of-the-axis, so to combine a pair of asymmetric TSAs into a symmetric structure is a natural idea as shown in Figure 13a [8]. Their resultant pattern in E-plane is just a 1-D saddleshaped beam in E-plane. In order to perform sharply slope-down, let the first (negative) side-lobe of one-half just coincide with the (positive) slope-down part of the main-lobe of another-half, as shown in Figure 13b.

A prototype of TSA (Figure 13c) with 1-D saddleshaped beam (Figure 13d) was designed as a feed of 1$\mathrm{D}$ reflect-array. Comparing with a traditional TSA feed with single peak in pattern, the 1-D aperture efficiency is increased from $70.6 \%$ up to $83.7 \%$ (corresponding

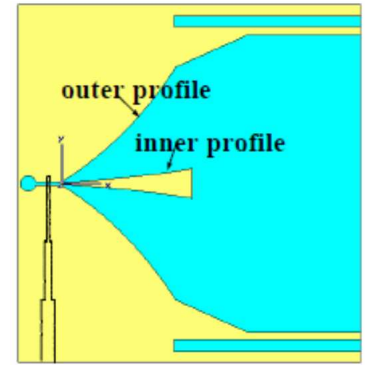

(a) Sketch

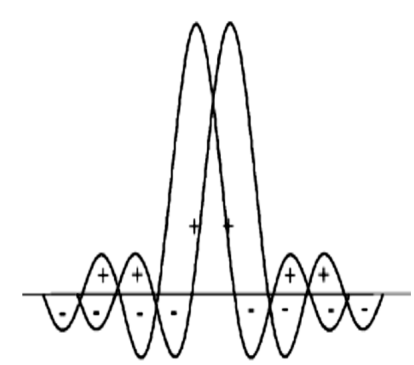

(b) Combination scheme of beams

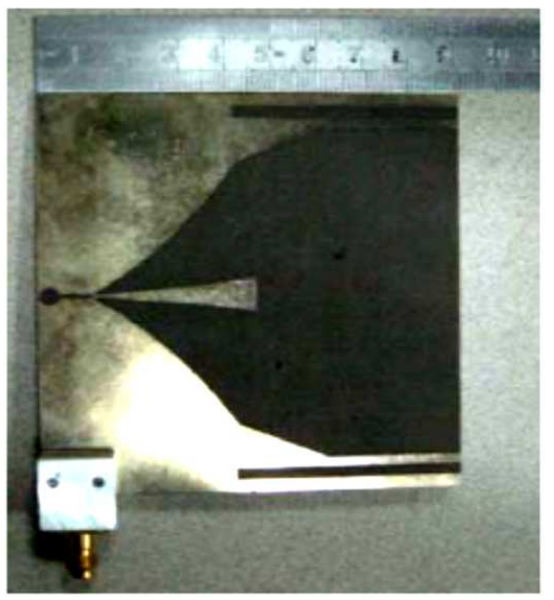

(c) Prototype

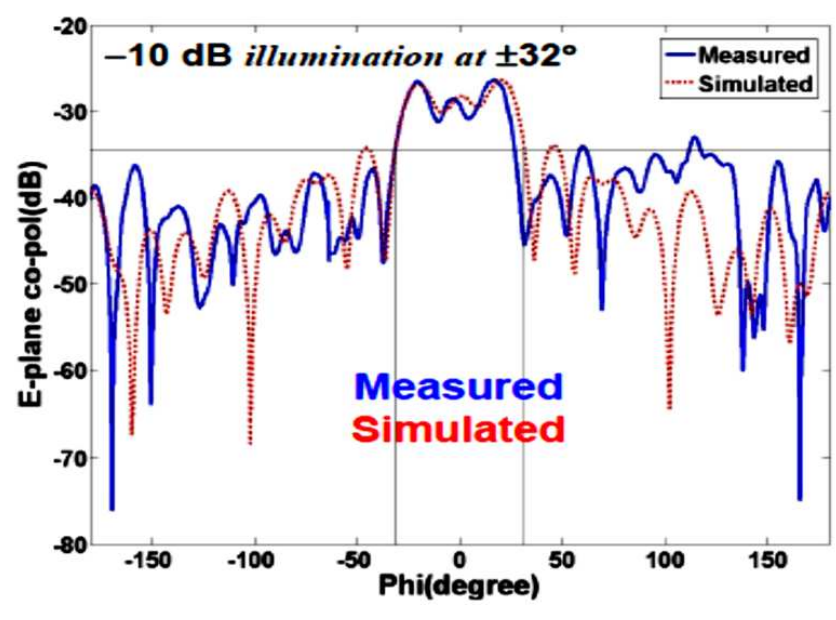

(d) 1-D saddle-shaped in E-plane

Figure 13. Beam-shaped TSA as a feed of reflect-array.

to the antenna efficiency increased from $56.5 \%$ up to $66.4 \%$ ). However, the phase-pattern of beam-shaped feed has about $\pm 28^{\circ}$ difference, it should be compensated by each element in reflect-array or transmit-array, but becomes unacceptable in a mirror reflector.

In the case of 2-D saddle-shaped beam-forming, two pieces of 1-D feed placed with appropriate out-ofthe axis in H-plane had been adopted (Figure 14a). However, the small intersected angle results in strong coupling between them, but larger intersected angle forms a wider saddle in beam (Figure 14b), which can be only used for a elliptical aperture of array. 


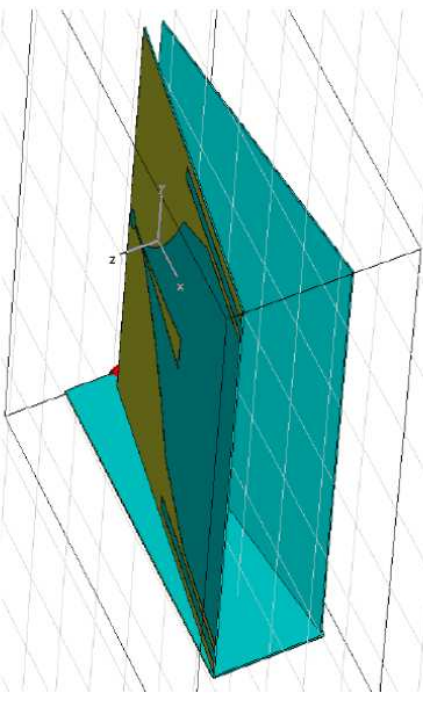

(a) Sketch

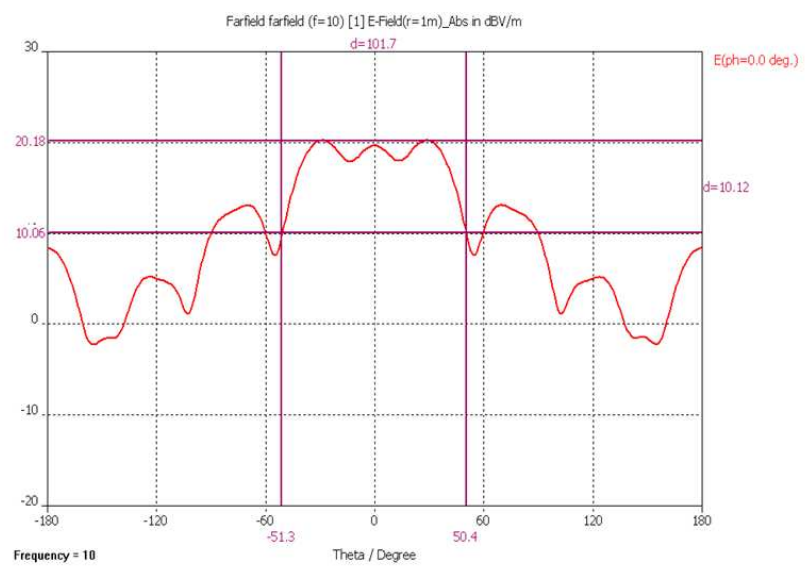

(b) Second 1-D saddle-shaped beam in H-plane

Figure 14. 2-D beam-shaped TSA as a feed of reflect-array.

\section{Coupled TSA for Monopulse Beam \\ FORMING}

Actually, early than developing a TSA with saddleshaped beam, a similar structural scheme was proposed to compose a slightly broader beam with single-peak in E-plane for making equalization to the beam-width in H-plane [9]. This structure can be considered as coupled TSA (C-TSA) fed by a pair of coupled slotlines in even-mode excitation as shown in Figure 15a, and then radiates a sum-beam ( $\Sigma$-beam). Hence, a reasonable deduction was led to odd-mode excitation if the slot-line pair is considered as a CPW as shown in Figure $15 \mathrm{~b}$, then to form a difference-beam ( $\Delta$-beam).

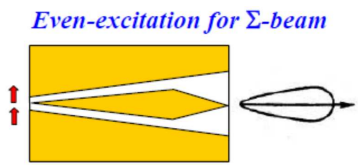

(a) Co-phased feeding

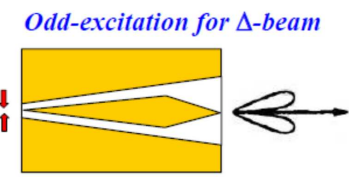

(b) Counter-phased feeding
Figure 15. Different feeding and patterns of C-TSA
However, in a monopulse radar system, which transmit $\Sigma$-beam and receive both $\Sigma$ - \& $\Delta$-beam should be simultaneously, a printed duplexer as hybrid network is necessary. Successively, a $1-\mathrm{D}$ and a $2-\mathrm{D}$ printed $\Sigma / \Delta$ duplexer [10] for Ka-band was developed.

The performances of 1-D duplexer (Figure 16): both $V S W R \leq 2: 1$ and isolation between $\Sigma$ - \& $\Delta$ - ports $\geq 35 \mathrm{~dB}$ within $(34.5 \sim 40.0) \mathrm{GHz}$ have been simulated and then verified by test.

The performances of 2-D duplexer (Figure 17): the $V S W R \leq 1.5: 1$ in simulation but $\leq 2.5: 1$ (at $\Delta_{E}$-port) \& $3.5: 1\left(\Delta_{H}\right.$-ports) within $(33 \sim 40) \mathrm{GHz}$ in test; the isolation for $\left[\Sigma-\Delta_{E}\right] \geq 40 \mathrm{~dB}$ and $\left[\Sigma-\Delta_{H}\right] \geq 27 \mathrm{~dB}$ within $(28 \sim 40) \mathrm{GHz}$ for both simulation and test.

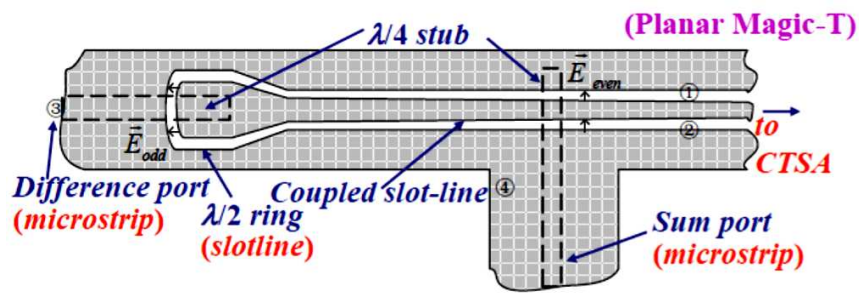

Figure 16. 1-D printed $\Sigma / \Delta$ duplexer for feeding C-TSA.

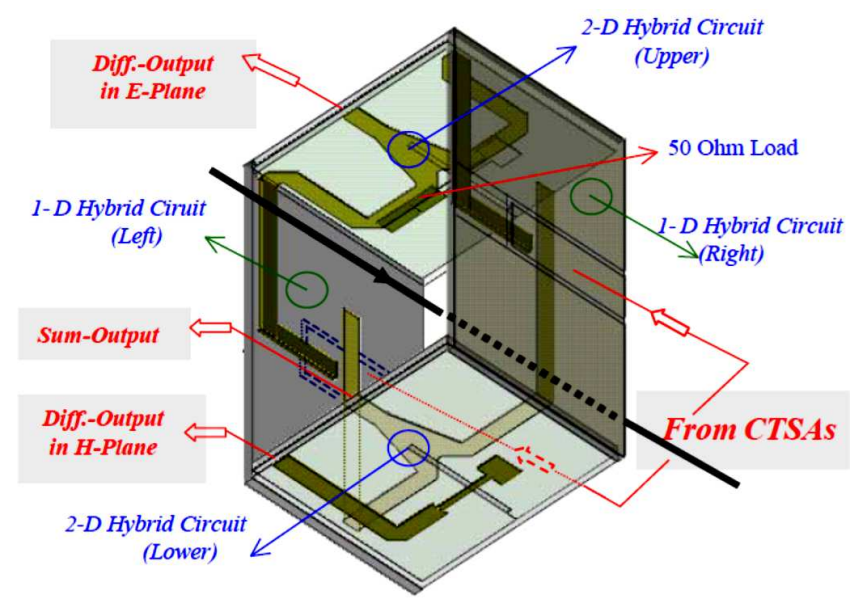

Figure 17. 2-D printed $\Sigma / \Delta_{E} \& \Delta_{H}$ duplexer for feeding C-TSA pair.

In view of the contradiction between gain of $\Sigma$-beam and null-depth of $\Delta$-beam needs to be coordinated, that means the configuration of C-TSA should be designed by optimization with multiple-objectives (gain and VSWR for $\Sigma$-state, null-depth and VSWR for $\Delta$ state). Thus, the method of moments was adopted in analysis program $[11,12]$ and the genetic algorithm was employed for optimization [13, 14]. An optimized CTSA combined with a 1-D duplexer for $(35 \sim 40) \mathrm{GHz}$ is described in Figure 18, its typical patterns at central frequency $37.5 \mathrm{GHz}$ are shown in Figure 19 too. 


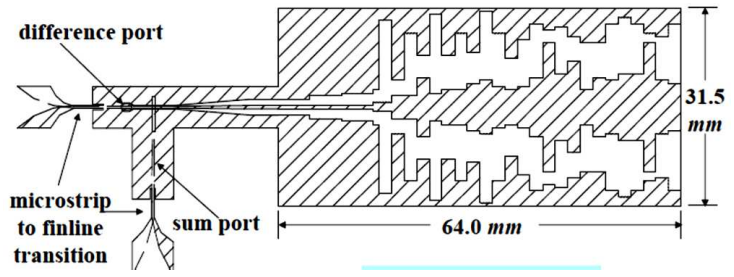

Figure 18. Optimized C-TSA for $\Sigma-/ \Delta$ - beams coordination.
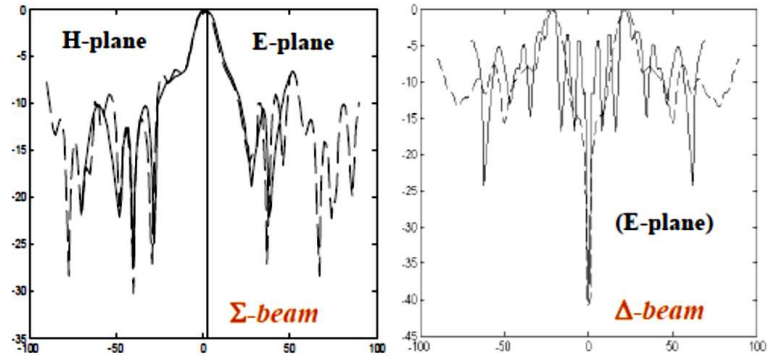

Figure 19. $\quad \Sigma-/ \Delta$ - beams of optimized C-TSA

[solid: tested / dot: simulated]

\section{Conclusion}

This article briefly reports several varietal tapered slotline antennas with individual features and applications, where the initial idea, structural scheme, simulated and measured results are explained for each one. More detailed information may be found from relative references.

\section{ACKNOWLEDGEMENT}

This work was supported by National Natural Science Fund of China (No. 69871006; No. 60371026; No. 60621002) and National High-tech. Project (under Grant 2007AA01Z264); and contributed by many graduates as listed in the references and others.

\section{REFERENCES}

[1] P. J. Gibson, "The vivaldi areial," in Proc. of 9th European Microwave Conference, Brighton, UK, 1979, pp. 101-105.

[2] S. N. Prasad and S. Mahapatra, "A novel MIC slot-line antenna," in Proc. of 9th European Microwave Conference, Brighton, UK, 1979, pp. 120-124.

[3] P. Zhang and W. X. Zhang, "Improved tapered slotline antennas by using grating loading," Microwave and Optical Technology Letters, vol. 52, no. 3, pp. 728-731, 2010, also China Patent No. ZL 200810156536.7.

[4] K. S. Yngvesson, T. L. Korzeniowski, and Y. S. Kim, "The tapered slot antenna - a new integrated element for millimeter-wave applications," IEEE Trans. On Microwave Theory and Techniques, vol. 37, no. 2, pp. 365-374, Mar. 1989.

[5] P. Zhang and W. X. Zhang, "Ultra-wideband bilateral tapered slot-line antenna fed by coplanar waveguide," Journal of Southeast University (English Edition), vol. 27, no. 2, pp. 128-131, 2011, also China Patent No. ZL 2010 10109810.2.

[6] W. Shen and W. X. Zhang, "Pattern synthesis of nonsymmetric tapered slotline antenna," Electronics Letters, vol. 42, no. 8, pp. 443-444, 2006, also China Patent No. ZL 200610037889.6.

[7] P. Zhang, W. X. Zhang, and S. J. Tang, "Improved tapered slot-line antennas loaded by grating," Journal of Electromagnetic Waves and Applications, vol. 23, no. 8/9, pp. 1039-1048, 2009, also China Patent No. ZL 20082 0215959.7.
[8] P. Zhang and W. X. Zhang, "Design.of an optimized feed for printed reflectarray antenna," in 4th International Workshop on Antenna Technology, Chiba, Japan, 2008, pp. 259-262.

[9] W. X. Zhang and X. H. Yang, "Research on coupled tapered slot-line antennas," in Proc.of IEE 7-th International Conference on Antennas and Propagation, UK, 1991, pp. 580-583.

[10] M. Yin, K. Kang, and W. X. Zhang, "A sum/difference-beams duplexer for the coupled tapered slot-line antenna," Electronics Letters, vol. 36, no. 11, pp. 935-936, 2000, also China Patent No. ZL 01-1 13577.8.

[11] K. Kang, M. Yin, and W. X. Zhang, "Coupled tapered slot-line antenna with sum/difference beams," in Proc. of Asia-Pacific Microwave Conference, Sydney, Australia, Dec. 2000, pp. 1136-1138.

[12] K. Kang and W. X. Zhang, "Method of moments analysis for sum/ difference beams of coupled tapered slot-line antennas," Journal of Microwaves, vol. 16, no. 1, pp. 6-12, 2000, [in Chinese].

[13] K. Kang, W. X. Zhang, and J. J. Li, “Optimisation of coupled tapered slot-line antenna for sum/difference beams," Electronics Letters, vol. 37, no. 9, pp. 548-549, 2001.

[14] K. Kang and W. X. Zhang, "application of multiobjective optimization methods to the printed antenna with sum/difference beams," Chinese J.of Radio Science, vol. 17, no. 5, pp. 485-489, 2002, [in Chinese].

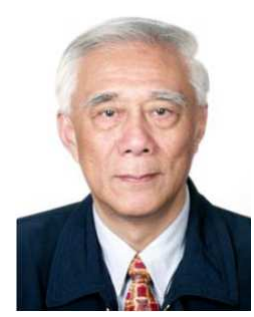

Wen-Xun Zhang was graduated in Radio Engineering from Nanjing Institute of Technology (NIT), and served in NIT/SEU (Southeast University) for 55 years. He is an emeritus professor, supervisor of Doctoral candidates, and was a deputy chair of academic steering committee to the State Key Laboratory of Millimeter Waves in China.

Zhang's main professional areas of interest include the engineering electromagnetism and antennas. He had published (authored or coauthored) some 470 papers in the international and national journals and conference proceedings. Zhang had written three famous books "Differential Equations for Radio Engineering" (1982, in Chinese); "Functional Methods for Electromagnetic Engineering" (1985, in Chinese); and also "Engineering Electromagnetism: Functional Methods" (1991, 1993, in English). He is a translator for the Chinese Edition of "ANTENNASfor all applications" (authored by J.D. Kraus \& R.J. Marhefka). He owns 12 patents on the printed antennas. Zhang has been member of Editorial Board of "Journal of Electromagnetic Waves and Applications", "Electromagnetics", "Microwave and Optical Technology Letters", "Waves in Random and Complex Media", "ECTI Transactions on Electrical Emgineering, Electronics and Communications", "REV Journal on Electronics and Communications", and "Chinese Journal of Radio Sciences".

Zhang is a Fellow of the Chinese Institute of Electronics (CIE), and served as Council member, a vice-president and then a consultant to Antenna Society. Zhang is a Fellow of the Institution of Engineering and Technology (IEE/IET) and a Chartered Engineer, and served as vice-chairman of Beijing Centre, a member of AP-PN Executive Team. Zhang is also a Fellow of the Institute of Electrical and Electronic Engineers (IEEE), and serves as a member of Transnational Committee of AP-Society, a past chairman of AP-MTT-EMC Joint Nanjing Chapter, which was the winner of the annual Best Chapter Awards $(1995,2000,2005)$ from AP-S and Most Improved Chapter Awards $(1995,1996,2005)$ from EMC-S. Zhang was an official member of the Commission B (Fields and Waves) and a chair of China (CIE) Committee to the International Union of Radio Science (URSI); was a member of Scientific Council to the Antenna Centre of Excellence (ACE) of Europe. Zhang is a Fellow of the Electromagnetics Academy; a Fellow of the Institute of Physics (IoP). He is also involved into various committees of international conferences and symposia, especially he had organized or chaired several International Symposia held in China. 